\title{
Vasculite na Hanseníase Mimetizando Doenças Reumáticas
}

\section{Vasculitis in Leprosy Mimicking Rheumatic Diseases}

\author{
Sandra Lúcia Euzébio Ribeiro ${ }^{(1)}$, Erilane Leite Guedes ${ }^{(2)}$, Helena Lúcia Alves Pereira ${ }^{(3)}$, Lucilene Sales de Souza ${ }^{(4)}$
}

\section{RESUMO}

Os autores alertam para o múltiplo espectro de apresentação clínica da hanseníase, destacando as lesões cutâneas necrosantes e manifestações articulares simulando doenças reumáticas, confundindo o diagnóstico e o tratamento correto. Nesses aspectos, são relatados dois casos do sexo feminino com hanseníase virchowiana e dimorfa cujas manifestações iniciais foram de lesões eritêmato-violáceas, necróticas e ulceradas, livedo reticular, oligoartrite, poliartralgias, mialgias e edema de membros inferiores, que mimetizaram algumas doenças reumáticas como o lúpus eritematoso sistêmico e a vasculite sistêmica (poliarterite nodosa).

Palavras-chave: hanseníase, fenômeno de Lúcio, vasculite sistêmica, doenças reumáticas.

\section{INTRODUÇÃO}

A hanseníase é uma doença infectocontagiosa crônica endêmica no Brasil, causada pelo Mycobacterium leprae, com afinidade por células do tecido cutâneo e de nervos periféricos $^{(1)}$. A partir de variações na resposta imune dos pacientes, pode evoluir para cura ou diferentes formas de apresentação clínica: indeterminada, tuberculóide, dimorfa ou virchowiana. Podem ainda ocorrer episódios inflamatórios agudos denominados "reações ou estados reacionais". As reações do tipo 1 ou reação reversa são mediadas pela imunidade celular e se apresentam clinicamente por neurite ou eritema e edema das lesões da pele; as do tipo 2 envolvem imunocomplexos ${ }^{(2)}$ e incluem o eritema nodoso hansênico, o eritema multiforme, o fenômeno de Lúcio e neurites ${ }^{(3)}$.

A artrite é uma manifestação bem conhecida na hanseníase, e sua freqüência nos relatos da literatura varia de $1 \%$ a $78 \%(4,5)$. Ocorre principalmente na vigência de episódios

\begin{abstract}
Leprosy has a large spectrum of clinical manifestations, including necrotizing skin lesions and joint complaints that sometimes mimic a primary rheumatic disease, confounding the correct diagnosis and treatment. Herein, the authors report two cases of women with leprosy, respectively virchowian and dimorphic forms, presenting initially with purple-reddish skin lesions, evolving with necrosis and ulceration, livedo reticularis, joint pain, oligoarthritis, myalgia, and leg edema. The differential diagnosis with systemic lupus erythematosus (SLE) and systemic vasculitis such as polyarteritis nodosa (PAN) is discussed.
\end{abstract}

Keywords: leprosy, Lucio's phenomenon, systemic vasculitis, rheumatic diseases.

reacionais, sobretudo na reação tipo $2^{(6)}$. Entretanto, artrite não relacionada aos quadros reacionais e em todas as formas da hanseníase foi descrita por vários autores ${ }^{(7-9)}$.

O fenômeno de Lúcio foi descrito pela primeira vez por Rafael Lucio e Ignácio Alvarado, em 1852, no México, e recebeu essa denominação em 1948 por Latapi e Zamo$\mathrm{ra}^{(10)}$. Representa uma variante da reação hansênica tipo 2 e histologicamente caracteriza-se como vasculite aguda necrosante, tendo como sinonímia a expressão "eritema necrosante"(3).

Apresentamos dois casos de hanseníase com lesão cutânea necrosante e quadro articular como manifestação inicial, mostrando o múltiplo espectro de apresentação clínica da doença, simulando doenças reumáticas.

\section{CASO 1}

MAF, 43 anos, sexo feminino, casada, doméstica, em dezembro de 1999 iniciou com astenia, anorexia, mialgias,

Recebido em 10/09/06. Aprovado, após revisão, em 23/01/07

Universidade Federal do Amazonas (UFAM), Hospital Universitário Getúlio Vargas (HUGV).

1. Professora do Departamento de Clínica Médica, Disciplina de Reumatologia da Universidade Federal do Amazonas (UFAM), pós-graduanda da Disciplina de Reumatologia da Universidade Federal de São Paulo (Unifesp)

2. Médica-residente da Disciplina de Clínica Médica da UFAM

3. Médica reumatologista assistente do Hospital Universitário Getúlio Vargas, Universidade Federal do Amazonas (UFAM), pós-graduanda da Disciplina de Reumatologia da Universidade Federal de São Paulo (Unifesp).

4. Médica dermatologista da Fundação Alfredo da Matta (FUAM)

Endereço para correspondência: Sandra Lúcia Euzébio Ribeiro, Avenida Apurimã, 4, Bairro Praça 14, CEP 69020-170, Manaus, AM, Brasil, telefone: (92) 3633-4977, e-mail: sandraeuzebio@vivax.com.br 
lesões cutâneas em face (Figura l) violáceas e livedo reticular, evoluindo com pápulas e pústulas em ambas hemifaces, edema nos membros inferiores (MMII) com algumas áreas ulceradas e necróticas (Figuras 2 e 3 ). Na ocasião, fator antinuclear (FAN) em Hep-2 1:320, padrão pontilhado fino; anticardiolipina IgG 4,1 GPL e IgM 7,0 MPL. Foi iniciado prednisona $40 \mathrm{mg} /$ dia com diagnóstico presuntivo de lúpus eritematoso sistêmico (LES).

Em dezembro de 2000, não havendo melhora, foi encaminhada ao dermatologista e submetida à biópsia das lesões com a hipótese diagnóstica de vasculite a esclarecer, mantida prednisona. A paciente relatou, ainda, ser portadora de hipertensão arterial sistêmica e de diabetes melito.

Em janeiro de 2001, retorna com o laudo da biópsia virchowiana reacional: infiltrados de histiócitos e polimor-

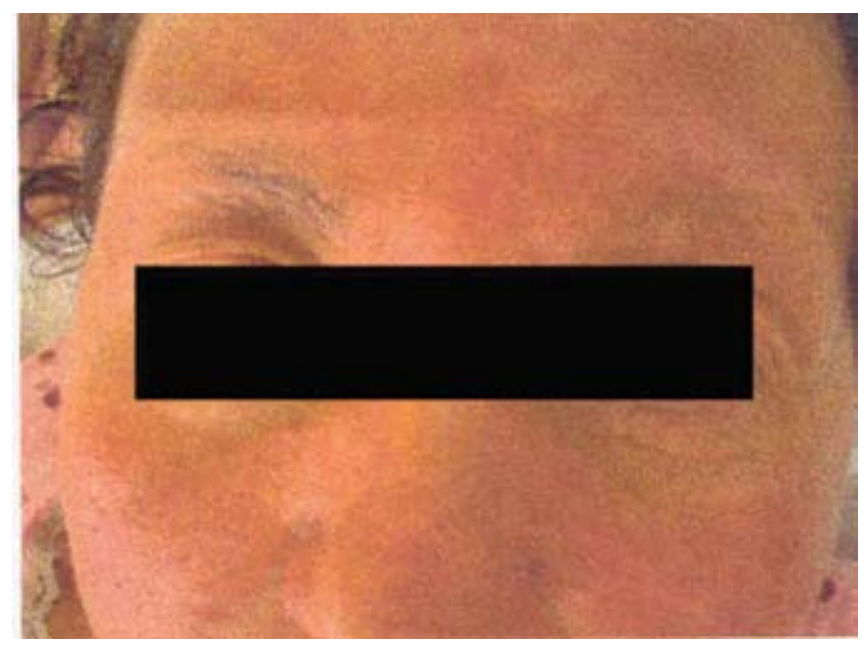

Figura 1 - Rash cutâneo facial.

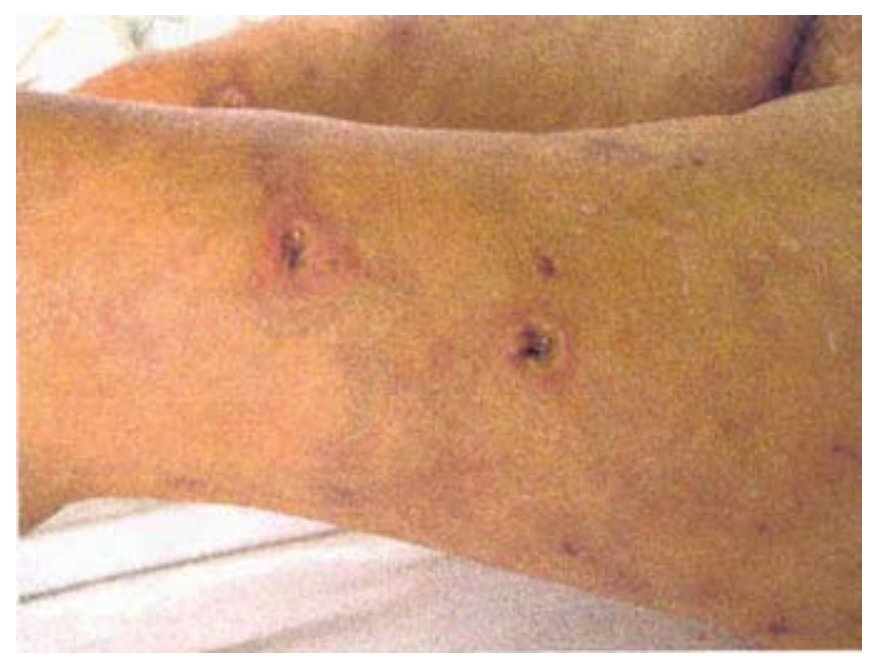

Figura 2 - Lesões ulceradas e necróticas em MMII.

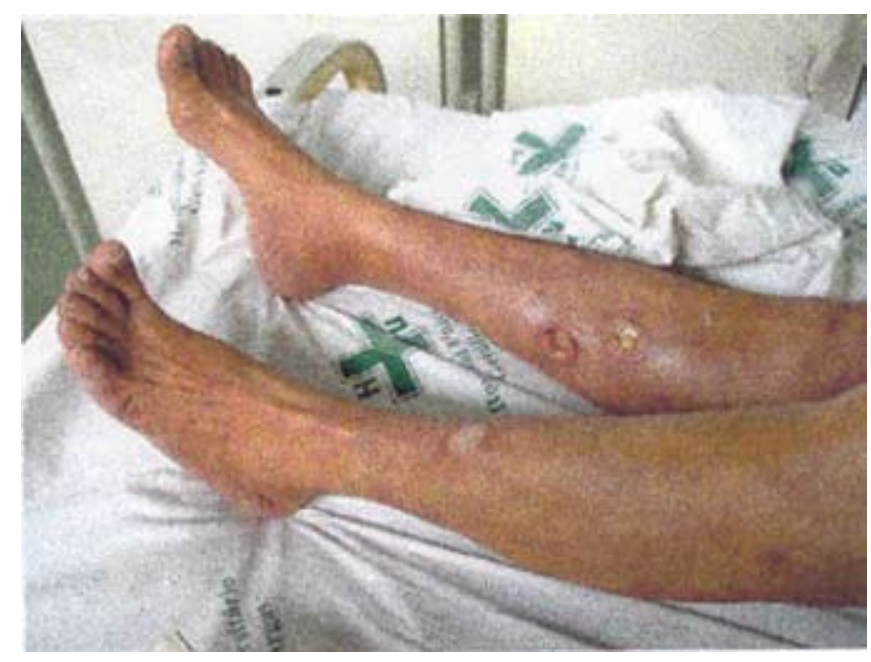

Figura 3 - Lesões ulceradas e necróticas em MMII.

fos nucleares, numerosos bacilos álcool-ácido-resistente (BAAR) íntegros e fragmentados, isolados e em globias. A pesquisa de BAAR na linfa foi positiva. Ao exame físico, nota-se importante lesão ulcerada em abdômen (Figura 4), com infecção secundária associada, várias lesões necróticas e ulceradas em membros superiores (MMSS), MMII e madarose bilateral. Foi iniciado tratamento específico para hanseníase multibacilar-PQT-MB-24 meses (dapsona $100 \mathrm{mg} /$ dia; rifampicina $600 \mathrm{mg} / \mathrm{mês}$; clofazimina $300 \mathrm{mg} / \mathrm{mês}$ e $50 \mathrm{mg} / \mathrm{dia}$ ) e solicitados exames. Na segunda consulta, retornou apresentando melhora do quadro de livedo reticular e do eritema facial, persistindo a lesão ulcerada com áreas de tecido granulomatoso em abdômen e as lesões violáceas em MMII. Estabeleceu-se o diagnóstico de hanseníase virchowiana reacional com lesões cutâneas

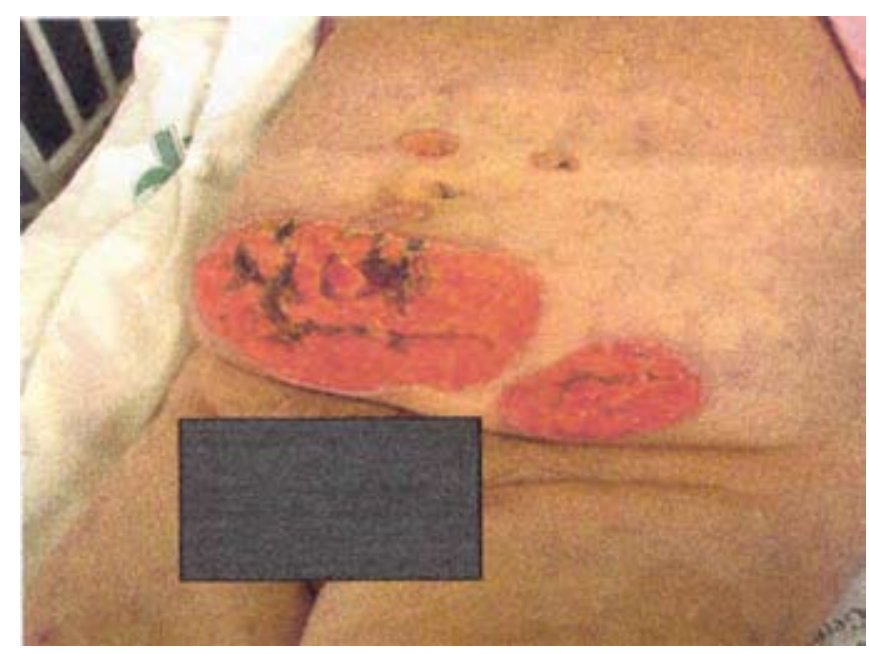

Figura 4 - Lesão com necrose e ulceração no abdômen. 
compatíveis com FL, mantida a terapia multidroga para hanseníase e a prednisona. Ao final de fevereiro foi observada melhora acentuada das lesões dos MMSS e MMII, da úlcera abdominal e desaparecimento do livedo reticular.

Em março de 2001, a paciente foi internada para o tratamento de pneumonia e esclarecimento das lesões necróticas e ulceradas, que haviam reaparecido. $\mathrm{Na}$ admissão, apresentava-se em regular estado geral, afebril; exame cardiovascular sem alterações; aparelho respiratório com murmúrio vesicular diminuído e estertores crepitantes em bases pulmonares; abdômen globoso com leve distensão, indolor à palpação, ausência de visceromegalias e presença de lesão ulcerada extensa com áreas de necrose em abdômen inferior, com algumas lesões ulceradas e necróticas; e edema em MMII. As úlceras abdominais estavam em lento processo de cicatrização, surgindo novas lesões ulceradas em região glútea direita. Laboratorialmente, apresentava anemia (hematócrito: 25,3\%; hemoglobina: $8,4 \mathrm{~g} / \mathrm{dl}$ ); velocidade de hemossedimentação (VHS): $56 \mathrm{~mm}$; glicemia: $212 \mathrm{mg} / \mathrm{dl}$, sem demais alterações laboratoriais. A radiografia de tórax apresentava imagem sugestiva de abscesso pulmonar de lobo médio direito. Iniciada antibioticoterapia; talidomida $200 \mathrm{mg} / \mathrm{dia}$ e aumentada a dose de prednisona $(40 \mathrm{mg} /$ dia para 80 $\mathrm{mg} /$ dia $)$ e clofazimina (100 mg/dia para $300 \mathrm{mg} /$ dia $)$. Com base nos dados clínicos, laboratoriais, na boa resposta à terapêutica instituída e com a segunda biópsia, com laudo de MHV-reacional: infiltrados de histiócitos e neutrófilos, numerosos BAAR granulosos, foi estabelecido o diagnóstico com fenômeno de Lúcio. Houve resolução completa do quadro infeccioso e gradual das lesões cutâneas, e a paciente recebeu alta hospitalar.

Em abril de 2001, após abandono do tratamento, foi novamente internada por recidiva das lesões cutâneas e infecção secundária associada, evoluindo com sepse que a levou ao óbito.

\section{CASO 2}

VCSC, 30 anos, sexo feminino, em fevereiro de 2000 iniciou artrite em tornozelos, poliartralgia simétrica em mãos, punhos, cotovelos, ombros, joelhos e pés, fraqueza muscular e parestesia em MMII, além dos sintomas gerais como astenia, anorexia, perda ponderal de aproximadamente $8 \mathrm{~kg}$. Negava febre. A paciente procurou o reumatologista, o qual solicitou exames e iniciou prednisona $40 \mathrm{mg} /$ dia. Porém, evoluiu com piora do quadro clínico, com livedo reticular, e surgiram máculas avermelhadas na face plantar do pé esquerdo, cianose de mãos e pés e dor intensa do $4^{\circ}$ e $5^{\circ}$ pododáctilos à esquerda.

Internada dia 30 de maio de 2000 para avaliação diagnóstica, com as hipóteses de poliarterite nodosa (PAN) e LES. Ao exame físico, apresentava-se em regular estado geral, afebril; abdômen, aparelho cardiovascular e respiratório sem alterações; pressão arterial de $130 / 90 \mathrm{mmHg}$; pulsos palpáveis e com boa amplitude; dor em mãos, punhos, tornozelos, pés, eritema violáceo em pés e mãos, cianose em polpas digitais com necrose de $4^{\circ}$ e $5^{\circ}$ pododáctilos à esquerda, diminuição da força muscular em MMII, principalmente à esquerda. A dose de prednisona foi aumentada (40 mg/dia para $80 \mathrm{mg} /$ dia) devido ao quadro de lesões isquêmicas e dor incapacitante. Sem melhora, optou-se pela realização de pulsoterapia com metilprednisolona $1 \mathrm{~g} /$ dia por três dias, com melhora parcial.

Exames laboratoriais: hematócrito 43\%; leucograma de $12.900 / \mathrm{mm}^{3}$; glicemia $81 \mathrm{mg} / \mathrm{dl}$; uréia $28 \mathrm{mg} / \mathrm{dl}$; creatinina $0,7 \mathrm{mg} / \mathrm{dl}$; VHS $58 \mathrm{~mm}$; anticardiolipina (IgG:3.0 GPL e IgM:1.5 MPL); anticoagulante lúpico, fator reumatóide (FR), FAN HEp-2, anti-DNA, anti-ENA, anti-SSA, anti-SSB: não-reagente; e sorologias para HIV, HbsAg, HVC e VDRL: todos negativos. Radiografia de tórax e ecocardiograma normal, ultra-sonografia abdominal (estudo de grandes vasos e artérias renais) com Doppler colorido não visualizando aneurismas. Realizados mielograma (sem alteração) e biópsia de pele e nervo sural. Com alta hospitalar em junho.

A paciente retorna somente em setembro de 2000 , usando prednisona $20 \mathrm{mg} /$ dia e carbamazepina $200 \mathrm{mg} /$ dia, referindo as mesmas queixas e com resultado das biópsias: pele (vasculite leucocitoclástica) e nervo sural (infiltrado inflamatório mononuclear e áreas de granulomas constituídos por histiócitos e linfócitos ao redor de áreas de necrose - neurite granulomatosa). Apesar de a coloração especial não ter evidenciado BAAR, a paciente foi encaminhada para avaliação dermatológica com hipótese de hanseníase.

Após avaliação com dermatologista em reunião clínica no Alfredo da Matta (centro de referência de hanseníase em Manaus-AM), foi estabelecido o diagnóstico de hanseníase dimorfa (MHBT) baseado no quadro clínico, exame neurológico (diminuição da força muscular em MMII, amiotrofia de perna mais acentuada à esquerda; nervos fibular comum e tibial posterior espessados bilateralmente; nervo radial e ulnar espessados à direita sem neurite, paresia da abdução do polegar à esquerda; dorsoflexão do pé à direita e extensão dos dedos), exames laboratoriais, pesquisa de BAAR em linfa negativa e nos resultados das biópsias, ini- 
ciando-se a poliquimioterapia multibacilar - PQT-MB-12 meses associada à prednisona $40 \mathrm{mg} /$ dia e a paciente foi encaminhada à fisioterapia.

Os sintomas permaneceram ainda por vários meses após a instituição da terapêutica, entretanto, por ocasião da alta, a paciente encontrava-se com remissão total da sintomatologia.

\section{DISCUSSÃO}

A hanseníase na forma virchowiana e dimorfa apresenta similaridades tanto clínicas como sorológicas com as doenças reumáticas, principalmente LES e artrite reumatóide (AR). Essas manifestações clínicas incluem desde lesões cutâneas (ulcerações, necrose isquêmica, vesículas e bolhas púrpura, nódulos e rash malar) até alterações sistêmicas, como hepatoesplenomegalia, artralgia e poliartrite. Entre as anormalidades sorológicas estão FR, FAN, anticorpo anticardiolipina (aCL), anticorpo anticitoplasma de neutrófilos (Anca), entre outros ${ }^{(11)}$.

O fenômeno de Lúcio representa reação cutânea necrosante grave que pode ocorrer na lepra de Lúcio, forma lepromatosa pura e primitiva, e em outras formas de hanseníase virchowiana ${ }^{(12)}$. Acomete, em geral, os pacientes não tratados, devido à ausência de diagnóstico ${ }^{(13)}$.

$\mathrm{Na}$ lepra de Lúcio, o fenômeno de Lúcio caracteriza-se por surtos de máculas eritematosas dolorosas, que evoluem com necrose central e posterior ulceração. As lesões acometem preferencialmente as extremidades, deixando cicatriz atrófica ${ }^{(10)}$.

$\mathrm{Na}$ hanseníase virchowiana, o fenômeno de Lúcio caracteriza-se por necrose em lesões de eritema nodoso ou de eritema polimorfo surgidos na evolução de uma reação hansênica, em pacientes com ou sem hansenomas (hanseníase virchowiana reacional não-nodular) ${ }^{(12)}$. Embora o Brasil ocupe o segundo lugar do mundo em números de casos de hanseníase, relatos de FL são $\operatorname{raros}^{(12,14,15)}$.

No eritema nodoso hansênico, a ulceração é rara, as lesões se localizam em face e membros, mas os sinais e sintomas sistêmicos são comuns e há a presença de poucos bacilos. As lesões podem ser também necrotizantes e graves, aumentando o risco de seqüelas neuromusculares. Já no $\mathrm{FL}$, a ulceração é a regra, não acomete o rosto, a sintomatologia geral é escassa ou inexistente e, na histopatologia, há proliferação endotelial, trombose, necrose isquêmica e um infiltrado mononuclear discreto e numerosos bacilos ao redor e nas paredes vasculares. As necroses cutâneas são atribuídas a tromboses vasculares induzidas pela invasão direta das paredes dos vasos e do endotélio pelos bacilos de Hansen. Porém, autores admitem que a leucocitoclasia poderia ocorrer na primeira fase da reação imunológica, não sendo evidenciável posteriormente ${ }^{(10)}$.

O eritema nodoso e as vasculites são as alterações cutâneas que mais comumente podem fazer parte do diagnóstico diferencial da hanseníase com as doenças reumáticas, podendo também encontrar outras manifestações menos freqüentes como fenômeno de Raynaud, livedo reticular, espessamento cutâneo, rash malar, edema periorbital ${ }^{(5,11,16)}$.

No primeiro caso relatado, as lesões cutâneas associadas à presença de auto-anticorpos direcionaram o diagnóstico inicial para o LES. Após biópsia de pele, confirmando o diagnóstico de hanseníase virchowiana reacional, foi instituída terapêutica apropriada (PQT-MB), porém a paciente permanecia com piora das lesões de pele e do estado geral, surgindo dúvida quanto à etiopatogenia das lesões cutâneas, ou seja, se o quadro estava relacionado à hanseníase com FL ou se havia associação de hanseníase e LES. A frequiência da associação de hanseníase e LES é baixa, talvez pela sobreposição clínico-laboratorial entre as duas patologias, segundo relatos da literatura ${ }^{(17,18)}$. Embora os auto-anticorpos sejam considerados marcadores de doenças auto-imunes, também têm sido detectados em doenças infecciosas, inclusive a hanseníase ${ }^{(19,20)}$. Há controvérsias na literatura quanto ao tratamento do FL; alguns autores acreditam que, por ser considerada uma forma reacional, deveria ser tratada com corticóide sistêmico e talidomida, mantendo o tratamento específico para hanseníase multibacilar ${ }^{(12,15)}$. Outros julgam que o tratamento deve ser o da hanseníase multibacilar (PQT-MB) acompanhado de cuidados locais ${ }^{(21)}$. Saoji et al..$^{(22)}$ verificaram que o tratamento com corticosteróide sistêmico não foi de grande benefício. Nessa paciente, após instituirmos a terapêutica com talidomida e aumentarmos a dose de prednisona e clofazimina, as lesões ulceradas começaram a apresentar sinais de cicatrização, conforme descrito na literatura ${ }^{(10,23)}$. Com o resultado da segunda biópsia e a boa resposta clínica ao tratamento, confirmou-se o diagnóstico de MHV com fenômeno de Lúcio.

A segunda paciente iniciou o quadro com manifestações sistêmicas, articulares e lesões necróticas de extremidades com VHS elevado, simulando o diagnóstico de LES ou poliarterite nodosa. Os exames laboratoriais não foram conclusivos, entretanto a biópsia do nervo sural evidenciando granulomas foi sugestiva de hanseníase. $\mathrm{O}$ que reforçou o diagnóstico da paciente foi espessamento dos nervos periféricos, observado posteriormente. Apesar de a paciente estar na quinta dose de tratamento (PQT-MB) e usando prednisona, 
ainda persistia com artrite e cianoses de extremidades, queixas não muito compatíveis com hanseníase.

O diagnóstico da hanseníase é relativamente simples, definido pela presença de alterações dermatoneurológicas (placas hipocrômicas com alteração da sensibilidade, espessamento neural) e baciloscopia positiva. As dificuldades e o erro de diagnóstico surgem particularmente nos pacientes em reação, nos quais as manifestações sistêmicas predominam, e os exames laboratoriais (VHS, FR e auto-anticorpos) podem estar alterados, assemelhando-se a doenças reumáticas.

Considerando a raridade das vasculites como forma de apresentação inicial na hanseníase e a dificuldade de diagnóstico desta e tendo em vista, também, que os exames complementares para vasculite não são específicos, mas apenas auxiliam na avaliação das funções orgânicas, devemos realizar biópsia do tecido clinicamente envolvido, que é essencial para o diagnóstico de vasculite ${ }^{(24)}$.

O uso de corticosteróide na hanseníase tem sua indicação nos quadros de reação, principalmente nas neurites em que o processo inflamatório pode ocasionar lesões

\section{REFERÊNCIAS}

1. Araújo MG: Hanseníase no Brasil. Rev Soc Bras Med Trop 36 (3): 373-82, 2003.

2. Foss NT: Hanseníase: aspectos clínicos, imunológicos e terapêuticos. An Bras Dermatol 74(2): 113-9, 1999.

3. Gilbert E, Cubria JL, Gratacos R, et al: Lepra de Lucio. Med Cutan Iber Lat Am 10: 41-6, 1982.

4. Modi TH, Lele RC: Acute joint manifestations in leprosy. J Assoc Physicians India 17: 247-77, 1969.

5. Albert D, Weisman MH, Kaplan R: The Rheumatic manifestations of leprosy. Medicine 59: 442-8, 1980.

6. Karat ABA, Karat S, Job CK, Furness MA: Acute exudative arthritis in leprosy-rheumatoid-like syndrome in association with erythema nodoso leprosum. BMJ 3: 770-2, 1967.

7. Lele RD, Sainan GS, Sharma KD: Leprosy presenting as rheumatoid arthritis. J Assoc Physicians India 13: 275-7, 1965.

8. Atkin SL, EL-Ghobarey A, Kamel M, Owen JP, Dick WC: Clinical and laboratory studies of arthritis in leprosy. BMJ 298: 1423-5, 1989.

9. Cossermelli-Messina W, Neto CF, Cossermelli W: Articular inflammatory manifestations in patients with different forms of leprosy. J Rheumatol 25: 111-9, 1988.

10. Pursley TV, Jacobson RR, Apisarnthanarax P: Lucio's Phenomenon. Arch Dermatol 116: 201-4, 1980.

11. Matthews LJ, Trautmam JR: Clinical and serological profiles in leprosy. The Lancet 915-8, 1965.

12. Pereira AC Jr: Hanseníase de Lúcio. An Bras Dermatol 68(1): 33-40, 1993.

13. Rea TH, Levan NE: Lucio's Phenomenon and diffuse nonnodular lepromatous leprosy. Arch Dermatol 114: 1023-8, 1978. neurológicas irreversíveis ${ }^{(2)}$. No segundo relato, a paciente apresentava comprometimento de vários troncos nervosos e, embora ainda sem diagnóstico definitivo de hanseníase, a corticoterapia não agravou a doença de base e provavelmente contribuiu na prevenção de seqüelas neurológicas, porém, na paciente com hanseníase multibacilar, a corticoterapia prolongada sem o tratamento específico pode ter contribuído para disseminação da hanseníase e as intercorrências infecciosas manifestadas pela paciente.

Os dois casos de hanseníase (virchowiana e dimorfa) iniciaram o quadro clínico de forma atípica, com manifestações articulares e vasculares assemelhando-se a doenças reumáticas, direcionando erroneamente o raciocínio clínico e retardando, assim, o diagnóstico e a terapêutica adequada.

Ao atendermos pacientes com vasculite sistêmica, devemos estar atentos para incidência de doenças infecciosas em nosso meio, considerando o caráter endêmico da hanseníase no Brasil.

Declaramos a inexistência de conflitos de interesse.

14. World Health Organization. Leprosy Elimination Project Status Report 2003 - Draft. Geneva: The World Health Organization, 2004.

15. Souza CS, Roselino AMF, Figueiredo F, Foss NT: Lucio's Phenomenon: Clinical and Therapeutic Aspects. Int J Lepr Other Mycobact Dis 68(4): 417-25, 2000.

16. Bonvoison B, Martin JM, Bouvier M, Bocquet B, Boulliat J, Duivon JP: Polyathrite Lépreuse; Deux observations (letter). Presse Méd 12: 302, 1983.

17. Posner DI, Guill MA: Coexistent Leprosy and Systemic Lupus Erythematosus. Cutis 39: 136-8, 1987.

18. Ferro RC, Fernandes SRM, Costallat LTL, Adilmuhib S: Lúpus Eritematoso Sistêmico Associado à moléstia de Hansen: Relato de três casos. Rev Bras Reumatol 31(1): 31-5, 1991.

19. Edington FLB, Bacellar MOAR, Machado PR, et al: Anticorpos Anticitoplasma de Neutrófilos (Anca) em Pacientes com Hanseníase. Jorn da Lig Reumatol Nort Nordest 2(1): 6-10, 2006.

20. Repka JCD, Skare TL, Salles G Jr, Paul GM: Anticorpos Anticardiolipina em pacientes com mal de Hansen. Rev Bras Reumatol 41(1): 1-6, 2001.

21. Azulay-Abulafia L, Spinelli L: Revendo a Hanseníase de Lucio e o Fenômeno de Lucio. Med Cutan Iber Lat Am 33(3): 125-33, 2005.

22. Saoji V, Salodkar A: Lucio Leprosy with Lucio phenomenon. Indian J Lepr 73(3): 267-72, 2001.

23. Saúl A, Novales J: La Lépre de Lucio-Latapí et le phénomène de Lucio. Acta Leprologica 1: 115-32, 1983.

24. Sato EI, Hatta FS, Castro CHM, Nunes DS, Santo BE: Avaliação multidisciplinar das vasculites sistêmicas num centro de atendimento terciário: experiência da Unifesp. Rev Bras Reumatol 38(3): 133-8, 1998. 